\title{
C. Homburg, S. Kuester, H. Krohmer (2009) Marketing Management - A Contemporary Perspective
}

\author{
McGraw-Hill, London et al. 2009, 1. Auflage, 642 Seiten, \\ ISBN-13 978-0-07-711724-5, 45,95€
}

\section{Sven Reinecke}

Die drei Autoren verfolgen mit diesem Lehrbuch zum Marketing-Management vier Ziele: Erstens soll das Werk das Gebiet des Marketing umfassend behandeln, d.h. sowohl das strategische, das operative und das sektorale Marketing als auch Aspekte der Marketingumsetzung abdecken. Zweitens wird eine theoretische Fundierung aller Teilgebiete angestrebt, die zusätzlich durch den Theorie- und Methodenanhang des Buchs ergänzt wird. Drittens wird eine internationale Orientierung verfolgt: Dies schlägt sich zum einen darin nieder, dass die Literaturbasis stark auf anerkannten internationalen wissenschaftlichen Marketingjournals beruht. Zum anderen werden Fallbeispiele und -studien aus Europa, Nordamerika und Asien präsentiert.

Viertens soll das Buch praxisorientiert ausgerichtet sein, indem aktuelles und relevantes Marketingwissen anwendungsbezogen dargestellt wird.

Es kann als äußerst ambitioniert angesehen werden, diese vier Ziele gleichzeitig zu verfolgen, zumal dabei durchaus Zielkonflikte bestehen.

Das Buch ist sehr klar, durchaus klassisch und nachvollziehbar aufgebaut. Nach einer kurzen Einführung werden im ersten Teil des Buchs strategische Aspekte des Marketingmanagements diskutiert. Dabei geht es sowohl um die Situationsanalyse als auch die Definition, Evaluation und Selektion von Marketingstrategien.

Der Marketing-Mix wird im zweiten Teil des Buchs präsentiert, wobei die Autoren begründet den Verkauf (Sales) an die Stelle des klassischen Instruments der Distribution (Place) stellen. Ergänzt wird dieser Teil durch einen umfassenden Abschnitt zum Kunden(beziehungs)management.

Der dritte Teil des Buchs widmet sich dem Marketing in spezifischen Anwendungskontexten und hebt somit Besonderheiten des Business-to-Business-, des Dienstleistungsund internationalen Marketing hervor.

Online publiziert: 07.08 .2009

(C) Gabler-Verlag 2009

Prof. Dr. S. Reinecke $(\square)$

Instituts für Marketing an der Universität St. Gallen (HSG), St. Gallen, Schweiz

E-Mail: Sven.Reinecke@unisg.ch 
Die Marketingimplementierung bzw. die organisatorische, technische und personalführungsbezogene Umsetzung des Marketing steht im Mittelpunkt des vierten Kapitels des Lehrbuchs.

Insgesamt handelt es sich bei dem Werk um ein durchaus klassisches englischsprachiges Lehrbuch zum Marketing-Management, das jedoch sowohl inhaltlich als auch methodisch besondere Akzente setzt.

Inhaltlich deckt das Buch tatsächlich alle relevanten Gebiete des Marketing-Managements ab, wobei es trotz des bereits beachtlichen Umfangs von über 640 Seiten nicht möglich ist, alle Themen zu vertiefen. Wie es sich für ein gutes Grundlagenwerk gehört, gilt in vielen Teilen der Grundsatz „Breite statt Tiefe“, wobei jedoch dem interessierten Leser immer Hinweise auf vertiefende Literatur geboten werden. Allerdings gelingt es dem Buch, spezifische Akzente zu setzen, die durchaus auch die Forschungsausrichtung der Autoren widerspiegeln. Das Thema Verkaufsmanagement wird ebenso wie das bedeutende Gebiet der Marketingimplementierung deutlich umfassender und kompetenter dargelegt als es in den meisten anderen Standardwerken des Marketing-Managements der Fall ist. Aktuell und für das Management wichtig sind ebenfalls die ausführlichen Ausführungen zum Behavioral Pricing. Vorbildcharakter haben ferner die Abschnitte zur Marketing- und Verkaufsorganisation sowie zum Personalmanagement. Auch die Diskussion der Marktorientierung aus dem Blickwinkel des Top-Managements und der anderen Funktionsbereiche (F\&E, Finanz- und Rechnungswesen usw.) ist innovativ und gelungen.

Werden in einem Grundlagenwerk ausgewählte Gebiete vertieft, so hat das zwangsläufig die Folge, dass andere Bereiche etwas weniger ausführlich behandelt werden können. So wird beispielsweise das Thema Direct Marketing extrem knapp dargestellt, was aber aufgrund des Fokusses auf Marketingmanagement durchaus nachvollziehbar ist und sinnvoll erscheint. Andererseits wird das gerade für das Management wichtige (aber leider oft ungeliebte) Gebiet der Marktforschung in dem Grundlagenwerk etwas stiefmütterlich behandelt; es wird nicht geschlossen behandelt, sondern in die verschiedenen Kapitel integriert und zum Teil in den Anhang verbannt. Ferner wäre es für eine sicherlich zu erwartende 2. Auflage auch sinnvoll, die management- und anwendungsrelevanten Themen Value Pricing, Preisdifferenzierung und Marketing Metrics etwas ausführlicher zu gewichten. Da zahlreiche Unternehmen große Herausforderungen bei der konsequenten Umsetzung der Marketingsegmentierung haben, wären auch hier weitere Implementierungshinweise wünschenswert. Der Untertitel des Buchs „A Contemporary Perspective“ lässt auch eine (noch) stärkere Berücksichtigung des Online-Zeitalters sowie des Themas „Marketing und Gesellschaftsverantwortung“ (Corporate Social Responsibility) erwarten.

Positiv hervorzuheben im „Konkurrenzumfeld der Marketingstandardwerke“ ist an dem vorliegenden Werk die konsequente theoretische Fundierung. Ferner ist es für ein englischsprachiges Buch mutig und gleichzeitig begrüßenswert, dass traditionelle Ansätze der deutschsprachigen Marketingwissenschaft (beispielsweise der Geschäftstypenansatz im B2B-Marketing von Backhaus et al.) nicht der Internationalität ,geopfert“ werden, sondern gleichberechtigt präsentiert werden - auch und gerade wenn das Buch weitgehend auf englischsprachigen Referenzen aufbaut. 
Das gleiche gilt für die Fallbeispiele und -studien, die tatsächlich nicht nur aus dem englischsprachigen Raum stammen, sondern aus den wichtigsten Wirtschaftsräumen der gesamten Welt. Hier ist ein guter Mix gelungen.

Aus didaktischer Sicht ist das Buch ebenfalls sehr überzeugend, auch wenn es etwas an das derzeit führende internationale Standardwerk im Marketingbereich erinnert. Zu Beginn jedes Kapitels werden gute Leitfragen definiert, die Studierende beim Lernen unterstützen. Sowohl die Zusammenfassungen am Kapitelende als auch die Stichwortliste sind für den Leser wertvoll. Ein weiteres Plus sind die umfassenderen Fallstudien, die auch von Dozierenden für Übungsveranstaltungen eingesetzt werden können.

Insgesamt enthält das Buch sehr viele anschauliche Grafiken, welche die erklärten Sachverhalte veranschaulichen. Das lesefreudige Layout wird lediglich durch die nicht ganz optimale Druckqualität mancher Abbildungen getrübt.

Insgesamt handelt es sich um ein Lehrbuch, das einen gelungenen Brückenschlag zwischen verschiedenen Welten bietet: zwischen Theorie und Praxis, zwischen deutschsprachiger Tradition und internationaler Orientierung sowie zwischen der Zielgruppe der Studierenden und jener von Führungskräften in der Wirtschaft. 